\title{
The Two-Body Momentum Distribution in Finite Nuclei
}

\author{
P. Papakonstantinou ${ }^{a}$, E. Mavrommatis ${ }^{a}$ and T.S. Kosmas ${ }^{b}$ \\ ${ }^{a}$ Physics Department, Division of Nuclear and Particle Physics, University of Athens, \\ GR-15771 Athens, Greece \\ ${ }^{b}$ Physics Department, Theoretical Physics Division, University of Ioannina, GR-45110 \\ Ioannina, Greece
}

\begin{abstract}
The two-body momentum distribution $\eta_{2}\left(\vec{p}_{1}, \vec{p}_{2}\right)$ of nuclei is studied. First, a compact analytical expression is derived for $Z=N$, $\ell$-closed nuclei, within the context of the independent particle shell model. Application to the light closed-shell nucleus ${ }^{16} \mathrm{O}$ is included and discussed. Next, the effect of dynamical, short-range correlations is investigated in the case of the light nucleus ${ }^{4} \mathrm{He}$, by including Jastrow-type correlations in the formalism. The effect is significant for large values of $p_{1}$ and $p_{2}$ and for angles between the vectors $\vec{p}_{1}$ and $\vec{p}_{2}$ close to $\gamma=180^{\circ}$ and $0^{\circ}$.
\end{abstract}

\section{Introduction}

The problem of short-range nucleon-nucleon correlations (SRC) in nuclei is still an open field in nuclear physics [1]. An indirect evidence for SRC was obtained by the observation of a depletion of valence shells in $\left(e, e^{\prime} p\right)$ reactions $[2,3]$. It is also well known that SRC modify the nucleon momentum distribution $[4,5]$, i.e. the Fourier transform of the one-body density matrix $\rho_{1}\left(\vec{r}_{1}, \vec{r}_{1^{\prime}}\right)$ in the variable $\vec{r}_{1}-\vec{r}_{1^{\prime}}$, by introducing significant contributions at values of momentum beyond the Fermi momentum. Experimental information from inclusive $\left(e, e^{\prime}\right)$ and exclusive $\left(e, e^{\prime} p\right)$ reactions established the existence of a high-momentum component in the momentum distribution.

Beyond the one-body density matrix and momentum distribution, rich information on the nuclear ground state and nucleon-nucleon correlations is contained in the two-body density matrix $(2 \mathrm{DM}) \rho_{2}\left(\vec{r}_{1}, \vec{r}_{2} ; \vec{r}_{1^{\prime}}, \vec{r}_{2^{\prime}}\right)$ and its various Fourier transforms in momentum space. Experimentally, exclusive two-nucleon knockout reactions can provide information on the relative behaviour of nucleon pairs embedded in the nuclear medium and give more direct insight into SRC $[6,7,8,9,10,11,12,13]$. A very important role is played by electron-nucleus scattering experiments with the availability of high-energy $100 \%$ duty cycle electron beams which made possible to carry out double coincidence $\left(e, e^{\prime} N N\right)$ experiments. Past, present and near future experiments at NIKHEF, MAMI and Jefferson Lab use ${ }^{3} \mathrm{He},{ }^{12} \mathrm{C}$ and ${ }^{16} \mathrm{O}$ as targets $[8,9,10,11]$.

The analysis of $\left(e, e^{\prime} 2 N\right)$ experiments is a complicated task [14, 15, 16, 17]. Among the most important processes of longitudinal character contributing to electromagnetically induced two-nucleon knockout is the scattering off a correlated nucleon pair followed by two-nucleon knockout during which each of the two nucleons interacts with the residual nucleus (final-state interactions - FSI). The minimal starting point in the analysis is the plane-wave impulse approximation (PWIA), in which the FSI are neglected and the twonucleon knock-out cross section can be expressed in terms of the two-hole spectral function $S\left(\vec{p}_{1}, \vec{p}_{2} ; E\right)$. The latter gives the joint probability of removing from the target two nucleons with momenta $\vec{p}_{1}$ and $\vec{p}_{2}$ leaving the residual nucleus with energy $E$ with respect to the target nucleus ground state. Under certain conditions it seems possible to extract information on the 2DM and its Fourier transforms and on nucleon-nucleon correlations. 
Taking the above into account, the development of a simple model of the ground-state $2 \mathrm{DM}$ in order to clarify the two-body characteristics of the nuclear ground state and the extent to which it is affected by the SRC appears useful.

Here we focus on the two-body momentum distribution (2bMD) $\eta_{2}\left(\vec{p}_{1}, \vec{p}_{2}\right)$, which is the Fourier transform of the 2DM $\rho_{2}\left(\vec{r}_{1}, \vec{r}_{2} ; \vec{r}_{1^{\prime}}, \vec{r}_{2^{\prime}}\right)$ in the variables $\vec{r}_{1}-\vec{r}_{1^{\prime}}$ and $\vec{r}_{2}-\vec{r}_{2^{\prime}}$. Another Fourier transform of the half-diagonal two-body density matrix $\rho_{2 h}\left(\vec{r}_{1}, \vec{r}_{2}, \vec{r}_{1^{\prime}}\right)=$ $\rho_{2}\left(\vec{r}_{1}, \vec{r}_{2} ; \vec{r}_{1^{\prime}}, \vec{r}_{2}\right)$ [18], namely the generalized momentum distribution $\eta(\vec{p}, \vec{Q})$, has been studied previously in the case of infinite nuclear matter [19] and finite nuclei [20]. The generalized momentum distribution plays an important role in the study of the FSI. The $2 \mathrm{bMD}$ is straighforwardly connected to the two-nucleon spectral function $S\left(\vec{p}_{1}, \vec{p}_{2} ; E\right)$ via an integration with respect to the energy (see Eq.(10) below). The two-nucleon spectral function in the case of infinite nuclear matter has been studied using correlated basis function perturbation theory including central and tensor correlations [21]. In the case of ${ }^{16} \mathrm{O}$ it has been evaluated including short-range correlations through defect functions calculated via the Bethe-Goldstone equation or correlation functions derived from variational calculations and long-range correlations using dressed RPA [15].

In this work, first an analytical expression is derived for the $2 \mathrm{bMD}$ of $Z=N, \ell$-closed nuclei in the independent-particle shell model with harmonic oscillator wave-functions and applied to the nucleus ${ }^{16} \mathrm{O}$. Our method is an extension of that developed in Refs. $[20,22,23,24]$ for the study of the nuclear form factor, the nuclear charge, matter and momentum distributions, the one-body density matrix and the generalized momentum distribution in closed shell nuclei. This calculation is expected to reproduce the main features of the $2 \mathrm{bMD}$, stemming from the finite nuclear size and Fermi statistics, being reliable at certain kinematical domains. By introducing the quantity $\eta_{2}\left(\vec{p}_{1}, \vec{p}_{2}\right) / \eta\left(\vec{p}_{1}\right) \eta\left(\vec{p}_{2}\right)$, where $\eta(\vec{p})$ is the nucleon momentum distribution, we pinpoint the effects of the finite size and of the statistical correlations.

Next, the effect of the SRC on the $2 \mathrm{bMD}$ of ${ }^{4} \mathrm{He}$ is studied by including Jastrow-type correlations in our calculation, via the lowest term of a cluster expansion. This so-called low order approximation (LOA) [25] has been exploited for the one-body density matrix and the two-body density matrix by Bohigas and Stringari [26] and Dal Rì, Stringari and Bohigas [27] and has been widely used to study single-particle nuclear properties, such as the (point-nucleon or charge) density [28, 29], the elastic or charge form factor $[26,27,29]$, the one-body density matrix in coordinate $[28,30]$ or momentum space [28] and the nucleon momentum distribution [26, 27, 28, 30], as well as two-body nuclear quantities, namely the two-body density matrix in coordinate space [31], the static structure function $S(Q)$ [27], the relative pair density distribution $\rho_{2}\left(r_{12}\right)[27,31$, the center-of-mass pair density distribution $\rho_{2}\left(R_{C M}\right)$ [31], the two-body relative momentum distribution $\eta_{2}\left(\vec{q}_{\text {rel }}\right)$ $[31,32]$, the two-body center-of-mass momentum distribution $\eta_{2}\left(\vec{P}_{\mathrm{CM}}\right)[31,32]$ and the combined two-body center-of-mass and relative momentum distribution $\eta_{2}\left(P_{\mathrm{CM}}, q_{\mathrm{rel}}\right)$ [32]. A two-gaussian central correlation function, $f(r)=1-c_{1} \exp \left(-r^{2} / \beta_{1}^{2}\right)+c_{2} \exp \left(-r^{2} / \beta_{2}^{2}\right)$, is used in this work. We find significant deviations from the independent-particle picture at high momenta, especially when the vectors $\vec{p}_{1}$ and $\vec{p}_{2}$ are antiparallel or parallel, as it is clearly shown by the values of the quantity $\eta_{2}\left(\vec{p}_{1}, \vec{p}_{2}\right) / \eta\left(\vec{p}_{1}\right) \eta\left(\vec{p}_{2}\right)$. There is, however, within the LOA, significant sensitivity of the results in some kinematical regions, mainly on the correlation function used to describe the SRC. For certain correlation functions one may obtain negative values of $\eta_{2}$ in few regions of momenta.

The definition and properties of the two-body momentum distribution are presented in detail in Section 2. In Section 3 an exact expression is derived for the $2 \mathrm{bMD}$ of $Z=N$, $\ell$-closed nuclei in the independent-particle shell model with harmonic oscillator wavefunctions and applied to the case of ${ }^{16} \mathrm{O}$. In Section 4 the effect of SRC on the $2 \mathrm{bMD}$ of ${ }^{4} \mathrm{He}$ is 
explored by including Jastrow-type correlations in our calculation via the LOA, and the results as well as the reliability of our approximation are discussed. Finally, in Section 5 we give a summary of the results and hints for possible future developments.

\section{The Two-Body Momentum Distribution}

The two-body momentum distribution $\eta_{2}\left(\vec{p}_{1}, \vec{p}_{2}\right)$ gives the combined probability density of finding in the nucleus a nucleon with momentum $\vec{p}_{1}$ and another one with momentum $\vec{p}_{2}$. In a system of $A(A \geq 2)$ identical particles, described by a unit-normalized state $|\Psi\rangle$, it can be defined as the expectation value

$$
\eta_{2}\left(\vec{p}_{1}, \vec{p}_{2}\right)=\left\langle\Psi\left|\sum_{\vec{s}, \overrightarrow{s^{\prime}}} a_{\vec{p}_{2}, \vec{s}^{\prime}}^{\dagger} a_{\vec{p}_{1}, \vec{s}}^{\dagger} a_{\vec{p}_{1}, \vec{s}} a_{\vec{p}_{2}, \overrightarrow{s^{\prime}}}\right| \Psi\right\rangle
$$

The $2 \mathrm{bMD}$ is the diagonal element of the $2 \mathrm{DM}$ in momentum space $\eta_{2}\left(\vec{p}_{1}, \vec{p}_{2} ; \vec{p}_{1^{\prime}}, \vec{p}_{2^{\prime}}\right)$,

$$
\eta_{2}\left(\vec{p}_{1}, \vec{p}_{2}\right)=\eta_{2}\left(\vec{p}_{1}, \vec{p}_{2} ; \vec{p}_{1}, \vec{p}_{2}\right)
$$

and may also be defined as the Fourier transform of the 2DM $\rho_{2}\left(\vec{r}_{1}, \vec{r}_{2} ; \vec{r}_{1^{\prime}}, \vec{r}_{2^{\prime}}\right)$ in the variables $\vec{r}_{1}-\vec{r}_{1^{\prime}}$ and $\vec{r}_{2}-\vec{r}_{2^{\prime}}$ :

$$
\eta_{2}\left(\vec{p}_{1}, \vec{p}_{2}\right)=\int \rho_{2}\left(\vec{r}_{1}, \vec{r}_{2} ; \vec{r}_{1^{\prime}}, \vec{r}_{2^{\prime}}\right) \mathrm{e}^{-i \vec{p}_{1} \cdot\left(\vec{r}_{1}-\vec{r}_{1^{\prime}}\right)} \mathrm{e}^{-i \vec{p}_{2} \cdot\left(\vec{r}_{2}-\vec{r}_{2^{\prime}}\right)} \mathrm{d}^{3} r_{1} \mathrm{~d}^{3} r_{2} \mathrm{~d}^{3} r_{1^{\prime}} \mathrm{d}^{3} r_{2^{\prime}}
$$

(By taking $\hbar=1$, the $2 \mathrm{bMD}$ has the dimension $(\text { length })^{6}$.) The normalization adopted is such that

$$
\int \eta_{2}\left(\vec{p}_{1}, \vec{p}_{2}\right) \mathrm{d}^{3} p_{1} \mathrm{~d}^{3} p_{2}=A(A-1)
$$

and the following sequential relation holds:

$$
\int \eta_{2}\left(\vec{p}_{1}, \vec{p}_{2}\right) \mathrm{d}^{3} p_{2}=(A-1) \eta\left(\vec{p}_{1}\right)
$$

where $\eta(\vec{p})$ is the one-body momentum distribution, normalized to the number of particles

$$
\int \eta(\vec{p}) \mathrm{d}^{3} p=A
$$

Equation (5) connects the $2 \mathrm{bMD}$ to the generalized momentum distribution $[19,20]$ $(\eta(\vec{p}, \vec{Q}=0)=(A-1) \eta(\vec{p}))$.

The $2 \mathrm{bMD} \eta_{2}\left(\vec{p}_{1}, \vec{p}_{2}\right)$ is related to the two-body center-of-mass momentum distribution $\eta_{2}\left(\vec{P}_{\mathrm{CM}}\right)\left(\vec{P}_{\mathrm{CM}}=\vec{p}_{1}+\vec{p}_{2}\right.$ is the two-nucleon center-of-mass momentum), and to the twobody relative momentum distribution $\eta_{2}\left(\vec{q}_{\text {rel }}\right)\left(\vec{q}_{\text {rel }}=\frac{1}{2}\left(\vec{p}_{1}-\vec{p}_{2}\right)\right.$ is the relative two-nucleon momentum) [31, 33]

$$
\begin{aligned}
& \eta_{2}\left(\vec{P}_{\mathrm{CM}}\right)=\int \eta_{2}\left(\vec{P}_{\mathrm{CM}} / 2+\vec{q}_{\mathrm{rel}}, \vec{P}_{\mathrm{CM}} / 2-\vec{q}_{\mathrm{rel}}\right) \mathrm{d}^{3} q_{\mathrm{rel}}, \\
& \eta_{2}\left(\vec{q}_{\mathrm{rel}}\right)=\int \eta_{2}\left(\vec{P}_{\mathrm{CM}} / 2+\vec{q}_{\mathrm{rel}}, \vec{P}_{\mathrm{CM}} / 2-\vec{q}_{\mathrm{rel}}\right) \mathrm{d}^{3} P_{\mathrm{CM}} .
\end{aligned}
$$

It is also related to the combined two-body center-of-mass and relative momentum distribution $\eta_{2}\left(P_{\mathrm{CM}}, q_{\mathrm{rel}}\right)[32]$

$$
\eta_{2}\left(P_{\mathrm{CM}}, q_{\mathrm{rel}}\right)=\int \eta_{2}\left(\vec{P}_{\mathrm{CM}} / 2+\vec{q}_{\mathrm{rel}}, \vec{P}_{\mathrm{CM}} / 2-\vec{q}_{\mathrm{rel}}\right) \mathrm{d} \Omega_{P_{\mathrm{CM}}} \mathrm{d} \Omega_{q_{\mathrm{rel}}} .
$$


Its relationship with the two-nucleon spectral function $S\left(\vec{p}_{1}, \vec{p}_{2} ; E\right)$ is

$$
\eta_{2}\left(\vec{p}_{1}, \vec{p}_{2}\right)=\int \mathrm{d} E S\left(\vec{p}_{1}, \vec{p}_{2} ; E\right)
$$

In a totally uncorrelated system the $2 \mathrm{bMD}$ is factorized as

$$
\eta_{2}\left(\vec{p}_{1}, \vec{p}_{2}\right)=\left(1-\frac{1}{A}\right) \eta\left(\vec{p}_{1}\right) \eta\left(\vec{p}_{2}\right)
$$

The constant $1-1 / A$ appears due to the normalization condition (4). For the ground state of a non-interacting Fermi system (a system of $A$ identical, non-interacting fermions) where only statistical correlations are present, starting from the 2DM [4], the 2bMD can be shown to have the form

$$
\eta_{2}\left(\vec{p}_{1}, \vec{p}_{2}\right)=\eta\left(\vec{p}_{1}\right) \eta\left(\vec{p}_{2}\right)-\frac{1}{\nu}\left|\eta_{1}\left(\vec{p}_{1}, \vec{p}_{2}\right)\right|^{2},
$$

where $\eta_{1}\left(\vec{p}_{1}, \vec{p}_{2}\right)$ is the one-body density matrix in momentum space and $\nu$ is the level degeneracy. Note that in the special case of a non-interacting Fermi system with $A=\nu$ Eq. (12) becomes identical with Eq. (11).

As an example we consider the ground state of an infinitely extended ideal Fermi gas (volume $\Omega$ and number of particles $A$ going to infinity while the particle density stays constant) with Fermi wave number $k_{\mathrm{F}}$ and degeneracy $\nu$. Equation (12) yields for the 2bMD per particle-pair $\tilde{\eta}_{2}^{\mathrm{F}}\left(\vec{p}_{1}, \vec{p}_{2}\right)=\eta_{2}^{\mathrm{F}}\left(\vec{p}_{1}, \vec{p}_{2}\right) /[A(A-1)]$

$$
\tilde{\eta}_{2}^{\mathrm{F}}\left(\vec{p}_{1}, \vec{p}_{2}\right)=\left(\frac{4}{3} \pi k_{\mathrm{F}}^{3}\right)^{-2} \theta\left(k_{\mathrm{F}}-p_{1}\right) \theta\left(k_{\mathrm{F}}-p_{2}\right)\left[1-\delta_{\vec{p}_{1} \vec{p}_{2}} / \nu\right]
$$

The momentum distribution per particle, $\tilde{\eta}^{\mathrm{F}}(p)=\eta^{\mathrm{F}}(p) / A=\eta^{\mathrm{F}}(\vec{p}) / A$, is given by

$$
\tilde{\eta}^{\mathrm{F}}(p)=\left(\frac{4}{3} \pi k_{\mathrm{F}}^{3}\right)^{-1} \theta\left(k_{\mathrm{F}}-p\right) .
$$

Let us define the quantity $\xi$ as a measure of finite-size effects, of statistical and short-range correlations of dynamical origin

$$
\xi\left(\vec{p}_{1}, \vec{p}_{2}\right) \equiv \eta_{2}\left(\vec{p}_{1}, \vec{p}_{2}\right) / \eta\left(\vec{p}_{1}\right) \eta\left(\vec{p}_{2}\right) .
$$

For an infinitely extended ideal Fermi gas $\xi$ is defined for $p_{1}, p_{2} \leq k_{F}$ and equals

$$
\xi^{\mathrm{F}}\left(\vec{p}_{1}, \vec{p}_{2}\right)=\left\{\begin{array}{ll}
1, & \vec{p}_{1} \neq \vec{p}_{2} \\
1-1 / \nu, & \vec{p}_{1}=\vec{p}_{2}
\end{array} .\right.
$$

From Eqs. (11) and (16) we realize that, in the case of the infinitely extended ideal Fermi system, if $\vec{p}_{1} \neq \vec{p}_{2}$, $\xi$ equals 1 . This holds even in the presence of dynamical SRC, if long-range order does not exist [34]. In the case of the infinitely extended ideal Fermi gas, $\xi=1-1 / \nu$ if $\vec{p}_{1}=\vec{p}_{2}$. As for the finite, non-interacting Fermi system, we realize from Eq. (12) that, if $\vec{p}_{1}=\vec{p}_{2}, \xi=1-1 / \nu$. Deviations of $\xi$ from this value show the effect of dynamical correlations. For $\vec{p}_{1} \neq \vec{p}_{2}$ deviations of $\xi$ from $1-1 / A$ is a measure of statistical and (or) dynamical correlations in a system of finite size. 


\section{Method of Calculation for $\ell$-Closed Nuclei - Ap- plication to ${ }^{16} \mathrm{O}$}

\subsection{Method of Calculation}

Let us now consider a system of $A$ identical non-interacting fermions in its ground state. The fermions occupy the lowest single-particle energy eigenstates $\left|n_{j}\right\rangle(j=1,2, \ldots, A / \nu)$ and the $2 \mathrm{bMD}$ is given by Eq. (12). In the case of the nucleus, $A$ is the mass number and $\nu$ is the degeneracy due to the nucleon spin and isospin. We consider $Z=N, \ell$-closed nuclei. In order to obtain closed analytical expressions for $\eta_{2}\left(\vec{p}_{1}, \vec{p}_{2}\right)$, we have assumed that the nucleons move in an isotropic harmonic oscillator potential and that the spin-orbit coupling is negligible, the center-of-mass and finite nucleon size corrections are small and the Coulomb interaction (relevant for protons) is small. Then Eq. (12) gives:

$$
\eta_{2}\left(\vec{p}_{1}, \vec{p}_{2}\right)=\frac{b^{6}}{\pi^{3}} \mathrm{e}^{-p_{1}^{2} b^{2}} \mathrm{e}^{-p_{2}^{2} b^{2}} \sum_{\mu_{1}=0}^{2 N_{\max }}\left(p_{1} b\right)^{\mu_{1}} \sum_{\mu_{2}=0}^{2 N_{\max }}\left(p_{2} b\right)^{\mu_{2}} \mathcal{G}_{\mu_{1} \mu_{2}}(\cos \gamma)
$$

where the coefficients $\mathcal{G}_{\mu_{1} \mu_{2}}$ are given by

$$
\mathcal{G}_{\mu_{1} \mu_{2}}(\cos \gamma)=4 f_{\mu_{1} / 2} f_{\mu_{2} / 2}-\sum_{\lambda_{1}=0 \lambda_{2}=0}^{\mu_{1}} \sum_{\mu_{2}}^{\mu_{2}} \mathcal{K}_{\lambda_{1} \lambda_{2}}(\cos \gamma) \mathcal{K}_{\left(\mu_{1}-\lambda_{1}\right)\left(\mu_{2}-\lambda_{2}\right)}(\cos \gamma)
$$

and the coefficients $f_{\mu / 2}$ and $\mathcal{K}_{\lambda_{1} \lambda_{2}}(\cos \gamma)$ are given by

$$
\begin{gathered}
f_{\mu / 2}=\sum_{n \ell, \text { occ. }} f_{n \ell}^{\mu / 2}, \\
\mathcal{K}_{\lambda_{1} \lambda_{2}}(\cos \gamma)=\sum_{n \ell, \text { occ. }} K_{n \ell}^{\lambda_{1} \lambda_{2}} P_{\ell}(\cos \gamma) .
\end{gathered}
$$

In the above equations $\gamma$ is the angle between the vectors $\vec{p}_{1}$ and $\vec{p}_{2}, \mathrm{~b}$ is the harmonic oscillator parameter, $P_{\ell}(x)$ is a Legendre polynomial and $N_{\max }=(2 n+\ell)_{\max }$ is the number

of energy quanta of the highest occupied $n \ell$-level. The coefficients $f_{n \ell}^{\mu / 2}, K_{n \ell}^{\lambda_{1} \lambda_{2}}$ are rational numbers that enter the corresponding expressions of $\eta(\vec{p})$ and $\eta_{1}\left(\vec{p}_{1}, \vec{p}_{1^{\prime}}\right)$ respectively. Analytical expressions for $f_{n \ell}^{\mu / 2}$ and $K_{n \ell}^{\lambda_{1} \lambda_{2}}$ can be found in Ref. [20]. They are different from zero only if the indices $\mu$ and $\lambda_{1}+\lambda_{2}$ are even.

The corresponding expression for the spherically symmetric nucleon momentum distribution $\eta(\vec{p})$ is $[22]$

$$
\eta(\vec{p})=\eta(p)=\frac{b^{3}}{\pi^{3 / 2}} \mathrm{e}^{-p^{2} b^{2}} \sum_{\lambda=0}^{N_{\max }}(p b)^{2 \lambda} 2 f_{\lambda} .
$$

It is verified that the $2 \mathrm{bMD} \eta_{2}\left(\vec{p}_{1}, \vec{p}_{2}\right)$ given by Eq. (17) satisfies the property of Eq. (5).

\subsection{Application to ${ }^{16} \mathrm{O}$}

Using the expressions (17) and (21), we obtain results for the quantities $\eta_{2}\left(\vec{p}_{1}, \vec{p}_{2}\right)$ and $\xi\left(\vec{p}_{1}, \vec{p}_{2}\right)$, in the case of the nucleus ${ }^{16} \mathrm{O}$. The harmonic oscillator parameter $b=1.7825 \mathrm{fm}$ has been determined so as to reproduce the experimental value of the charge root mean square radius of ${ }^{16} \mathrm{O},\langle r\rangle^{1 / 2}=2.737 \mathrm{fm}$ [35]. In Fig. 1 the $2 \mathrm{bMD}$ of ${ }^{16} \mathrm{O}$ in the harmonic oscillator model (continuous lines) is plotted for $\vec{p}_{1}$ parallel to $\vec{p}_{2}$ and for $p_{1}=0,1$ and 
$1.5 \mathrm{fm}^{-1}$, as a function of the variable $\mathrm{p}_{2}\left(\vec{p}_{2}=\mathrm{p}_{2} \hat{p}_{1}\right)$. In the same graphs the $2 \mathrm{bMD}$ of an infinite ideal Fermi gas is shown (dashed lines), calculated via Eq. (13) with $\nu=4$ and with Fermi wave number $k_{\mathrm{F}}$ equal to the one corresponding to ${ }^{16} \mathrm{O}$, namely $k_{\mathrm{F}}=1.1 \mathrm{fm}^{-1}$. This latter value has been calculated using local density approximation and coincides with the one used in Ref. [36]. In order to make the comparison meaningful, the 2bMD of the ideal Fermi gas was normalized to the same number of particle pairs as those found in ${ }^{16} \mathrm{O}$, namely to $A(A-1)=240$.

It is evident from Eq. (13) that the 2bMD of the ideal Fermi gas exhibits discontinuities at $p_{1,2}=k_{\mathrm{F}}$ and at $\vec{p}_{1}=\vec{p}_{2}$. This is observed in the first two panels of Fig. 1 $\left(p_{1}=0,1 \mathrm{fm}^{-1}\right)$ at $\mathrm{p}_{2}=p_{1}$ and at $\mathrm{p}_{2}= \pm k_{\mathrm{F}}$. In the case of the finite system ${ }^{16} \mathrm{O}$ in the harmonic oscillator model a rather similar behaviour is observed, but the 2bMD changes smoothly with $\mathrm{p}_{2}$. For $p_{1}=1.5 \mathrm{fm}^{-1}$ (which is higher than $k_{\mathrm{F}}=1.1 \mathrm{fm}^{-1}$ ) the $2 \mathrm{bMD}$ of the ideal Fermi gas has vanished and that of ${ }^{16} \mathrm{O}$ has dropped considerably.

In Fig. 2 the quantity $\xi\left(\vec{p}_{1}, \vec{p}_{2}\right)$ is plotted in the case of ${ }^{16} \mathrm{O}$ as a function of $\cos \gamma$ and for $p_{1}=p_{2}=0,1$ and $4 \mathrm{fm}^{-1}$. The values of $\xi$ lie between $0.75(=1-1 / \nu)$ and $1 . \xi$ is equal to 0.75 when $\vec{p}_{1}=\vec{p}_{2}$ (as shown for example in the first panel, and in the other two panels when $\cos \gamma=1)$. We realize that for high values of $p_{1}$ and $p_{2}, \xi$ tends to 1 and 0.75 for $\cos \gamma=0$ and $\cos \gamma=-1$ respectively. The deviation of the values of $\xi$ from 1 or 0.75 is a finite-size effect in the presence of statistical correlations. In the case when $p_{1} \neq p_{2}$ (not shown) the quantity $\xi$ as a function of $\cos \gamma$ remains higher than 0.75 and below or equal to 1 , following a similar behaviour as in Fig. 2, namely $\xi(-1)>\xi(1)$ and $\xi\left(x_{0}\right)=1$ for some value $\cos \gamma=x_{0}<0$.

\section{The Effect of Short-Range Correlations}

Up to now we have ignored the effect of dynamical, short-range correlations on the $2 \mathrm{bMD}$. It is expected that such a calculation is reliable only in restricted kinematical domains, namely for $p_{1}, p_{2} \leq k_{\mathrm{F}}$. In this Section we make an attempt to investigate the effect of the SRC by including Jastrow-type correlations in our study.

\subsection{Method of Calculation}

The approach adopted here is based on the Jastrow formalism with a state-independent central correlation function, which introduces the short range correlations. Employing the low-order approximation (LOA) of Ref. [27] for the 2DM and performing the spinisospin summation $(\nu=4)$ in Eq. (14) of Ref. [27], we obtain for the correlated 2DM $\rho_{2}^{\text {corr }}\left(\vec{r}_{1}, \vec{r}_{2} ; \vec{r}_{1^{\prime}}, \vec{r}_{2^{\prime}}\right)$ the following expression:

$$
\begin{aligned}
\rho_{2}^{\text {corr }} & \left(\vec{r}_{1}, \vec{r}_{2} ; \vec{r}_{1^{\prime}}, \vec{r}_{2^{\prime}}\right)=\left[1+g\left(r_{12}, r_{1^{\prime} 2^{\prime}}\right)\right] \rho_{2}\left(\vec{r}_{1}, \vec{r}_{2} ; \vec{r}_{1^{\prime}}, \vec{r}_{2^{\prime}}\right) \\
& +\int\left[g\left(r_{13}, r_{1^{\prime} 3}\right)+g\left(r_{23}, r_{2^{\prime} 3}\right)\right]\left[\rho_{1}\left(\vec{r}_{1}, \vec{r}_{1^{\prime}}\right) \rho_{2 h}\left(\vec{r}_{2}, \vec{r}_{3}, \vec{r}_{2^{\prime}}\right)\right. \\
& \left.-\nu^{-1} \rho_{1}\left(\vec{r}_{1}, \vec{r}_{2^{\prime}}\right) \rho_{2 h}\left(\vec{r}_{2}, \vec{r}_{3}, \vec{r}_{1^{\prime}}\right)-\nu^{-1} \rho_{1}\left(\vec{r}_{1}, \vec{r}_{3}\right) \rho_{2}\left(\vec{r}_{2}, \vec{r}_{3} ; \vec{r}_{2^{\prime}}, \vec{r}_{1^{\prime}}\right)\right] \mathrm{d}^{3} r_{3} \\
& -\nu^{-1} \iint g\left(r_{34}, r_{34}\right)\left\{\rho_{2 h}\left(\vec{r}_{2}, \vec{r}_{4}, \vec{r}_{3}\right) \rho_{2}\left(\vec{r}_{1}, \vec{r}_{3} ; \vec{r}_{1^{\prime}}, \vec{r}_{2^{\prime}}\right)\right. \\
& +\rho_{1}\left(\vec{r}_{1}, \vec{r}_{3}\right)\left[\rho_{1}\left(\vec{r}_{2}, \vec{r}_{2^{\prime}}\right) \rho_{2 h}\left(\vec{r}_{3}, \vec{r}_{4}, \vec{r}_{1^{\prime}}\right)-\nu^{-1} \rho_{1}\left(\vec{r}_{2}, \vec{r}_{1^{\prime}}\right) \rho_{2 h}\left(\vec{r}_{3}, \vec{r}_{4}, \vec{r}_{2^{\prime}}\right)\right. \\
& \left.\left.-\nu^{-1} \rho_{1}\left(\vec{r}_{2}, \vec{r}_{4}\right) \rho_{2}\left(\vec{r}_{3}, \vec{r}_{4} ; \vec{r}_{1^{\prime}}, \vec{r}_{2^{\prime}}\right)\right]\right\} \mathrm{d}^{3} r_{3} \mathrm{~d}^{3} r_{4},
\end{aligned}
$$

where $r_{i j}=\left|\vec{r}_{i}-\vec{r}_{j}\right|, g\left(r, r^{\prime}\right) \equiv f(r) f\left(r^{\prime}\right)-1$ and $f(r)$ is the correlation function, which has to obey the conditions $f(0)<1$ and for $r \rightarrow \infty, f(r) \rightarrow 1$. 
In Eq. (22) the one-body density matrix $\rho_{1}\left(\vec{r}, \overrightarrow{r^{\prime}}\right)$, the $2 \mathrm{DM} \rho_{2}\left(\vec{r}_{1}, \vec{r}_{2} ; \vec{r}_{1^{\prime}}, \vec{r}_{2^{\prime}}\right)$ and the half-diagonal $2 \mathrm{DM} \rho_{2 h}\left(\vec{r}_{1}, \vec{r}_{2}, \vec{r}_{1^{\prime}}\right)$ are calculated in the harmonic-oscillator model. The correlated $2 \mathrm{bMD}, \eta_{2}^{\operatorname{corr}}\left(\vec{p}_{1}, \vec{p}_{2}\right)$, is then calculated by Fourier transforming $\rho_{2}^{\text {corr }}\left(\vec{r}_{1}, \vec{r}_{2} ; \vec{r}_{1^{\prime}}, \vec{r}_{2^{\prime}}\right)$ according to Eq. (3). The correlated momentum distribution $\eta^{\operatorname{corr}}(\vec{p})$ is calculated likewise in the LOA by performing the spin-isospin summation in expression (13) of Ref. [27] for the one-body density matrix and by Fourier-transforming with respect to $\vec{r}_{1}-\vec{r}_{1^{\prime}}$.

The LOA preserves the normalization of the density matrices. As a consequence, the one- and two-body momentum distributions calculated in the LOA obey the normalization conditions (6) and (4), as well as the sequential relation (5). However, in the LOA some probability distributions (i.e. positive-definite quantities) may obtain negative values within regions of their domain. This problem is probably related to the $A$-representability of the LOA two-body density matrices. For example, it was encountered in calculations of the relative distribution function $\rho_{2}\left(r_{12}\right)$ [27] and the combined two-body center-of-mass and relative momentum distribution $\eta_{2}\left(P_{\mathrm{CM}}, q_{\mathrm{rel}}\right)$ [32], which implies that it may show up in the $2 \mathrm{bMD}$ too, since the latter is related to $\eta_{2}\left(P_{\mathrm{CM}}, q_{\mathrm{rel}}\right)$ via Eq. (9). In the case of the $\rho_{2}\left(r_{12}\right)$ the problem was subsequently solved by an appropriate modification of the correlation function [31]. The above remarks are highly relevant to the choice of the correlation function used in this work, as we will discuss in due course.

\subsection{Application to ${ }^{4} \mathrm{He}$}

The 2bMD and the momentum distribution of ${ }^{4} \mathrm{He}$ in the harmonic oscillator model, which we will hereafter denote by $\eta_{2}^{0}\left(\vec{p}_{1}, \vec{p}_{2} ; b\right)$ and $\eta^{0}(p ; b)$ respectively, are given by (see Eqs. (17), (21))

$$
\begin{aligned}
\eta_{2}^{0}\left(\vec{p}_{1}, \vec{p}_{2} ; b\right) & =\frac{12 b^{6}}{\pi^{3}} \mathrm{e}^{-b^{2}\left[p_{1}^{2}+p_{2}^{2}\right]}, \\
\eta^{0}(p ; b) & =\frac{4 b^{3}}{\pi^{3 / 2}} \mathrm{e}^{-p^{2} b^{2}} .
\end{aligned}
$$

yielding $\xi\left(\vec{p}_{1}, \vec{p}_{2}\right)=0.75$. The case falls into the category of non-interacting Fermi systems with $A=\nu$, hence Eq. (11) holds (see remark under Eq. (12)). The harmonic oscillator parameter $b=1.382 \mathrm{fm}$ reproduces the experimental value of the charge root mean square radius of ${ }^{4} \mathrm{He},\left\langle r^{2}\right\rangle^{1 / 2}=1.67 \mathrm{fm}$ [35] in this model (corrections due to center-of-mass motion and finite nucleon size are taken into account). We include SRC in the LOA using a twogaussian $(2 \mathrm{G})$ correlation function, $f(r)=1-c_{1} \exp \left(-r^{2} / \beta_{1}^{2}\right)+c_{2} \exp \left(-r^{2} / \beta_{2}^{2}\right)$. The expression of the $2 \mathrm{bMD}$ is

$$
\begin{aligned}
& \eta_{2}^{\operatorname{corr}}\left(\vec{p}_{1}, \vec{p}_{2}\right)=\eta_{2}^{0}\left(\vec{p}_{1}, \vec{p}_{2} ; b\right)+\delta \eta_{2}\left(\vec{p}_{1}, \vec{p}_{2} ; b, c_{1}, y_{1}\right)+\delta \eta_{2}\left(\vec{p}_{1}, \vec{p}_{2} ; b,-c_{2}, y_{2}\right) \\
- & 24 c_{1} c_{2} \frac{b^{6}}{\pi^{3}}\left\{\frac{1}{\left[\left(1+4 y_{1}\right)\left(1+4 y_{2}\right)\right]^{3 / 2}} \mathrm{e}^{-b^{2}\left[\frac{1}{2}\left(\frac{1+2 y_{1}}{1+4 y_{1}}+\frac{1+2 y_{2}}{1+4 y_{2}}\right)\left(p_{1}^{2}+p_{2}^{2}\right)+\left(\frac{2 y_{1}}{1+4 y_{1}}+\frac{2 y_{2}}{1+4 y_{2}}\right) \vec{p}_{1} \cdot \vec{p}_{2}\right]}\right. \\
+ & \frac{2}{\left(1+3 y_{1}+3 y_{2}+8 y_{1} y_{2}\right)^{3 / 2}}\left[\mathrm{e}^{-\frac{1+2 y_{1}+2 y_{2}}{1+3 y_{1}+3 y_{2}+8 y_{1} y_{2}} p_{1}^{2} b^{2}} \mathrm{e}^{-p_{2}^{2} b^{2}}+\left(p_{1} \leftrightarrow p_{2}\right)\right] \\
- & \left.\frac{5}{\left(1+2 y_{1}+2 y_{2}\right)^{3 / 2}} \mathrm{e}^{-b^{2}\left(p_{1}^{2}+p_{2}^{2}\right)}\right\}
\end{aligned}
$$

where $y_{i} \equiv b^{2} / \beta_{i}^{2}$ and

$$
\begin{aligned}
\delta \eta_{2}\left(\vec{p}_{1}, \vec{p}_{2} ; b, c, y\right) & \equiv \frac{12 b^{6}}{\pi^{3}}\left\{\left[\frac{10 c}{(1+2 y)^{3 / 2}}-\frac{5 c^{2}}{(1+4 y)^{3 / 2}}\right] \mathrm{e}^{-b^{2}\left(p_{1}^{2}+p_{2}^{2}\right)}\right. \\
& -\frac{2 c}{(1+4 y)^{3 / 2}} \mathrm{e}^{-b^{2}\left[\frac{1+3 y}{1+4 y}\left(p_{1}^{2}+p_{2}^{2}\right)+\frac{2 y}{1+4 y} \vec{p}_{1} \cdot \vec{p}_{2}\right]}
\end{aligned}
$$




$$
\begin{aligned}
& +\frac{c^{2}}{(1+4 y)^{3 / 2}} \mathrm{e}^{-b^{2}\left[\frac{1+2 y}{1+4 y}\left(p_{1}^{2}+p_{2}^{2}\right)+\frac{4 y}{1+4 y} \vec{p}_{1} \cdot \vec{p}_{2}\right]} \\
& +\frac{2 c^{2}}{[(1+2 y)(1+4 y)]^{3 / 2}}\left[\mathrm{e}^{-\frac{1}{1+2 y} p_{1}^{2} b^{2}} \mathrm{e}^{-p_{2}^{2} b^{2}}+\left(p_{1} \leftrightarrow p_{2}\right)\right] \\
& \left.-\frac{4 c}{(1+3 y)^{3 / 2}}\left[\mathrm{e}^{-\frac{1+2 y}{1+3 y} p_{1}^{2} b^{2}} \mathrm{e}^{-p_{2}^{2} b^{2}}+\left(p_{1} \leftrightarrow p_{2}\right)\right]\right\}
\end{aligned}
$$

The corresponding expression for the momentum distribution $\eta^{\text {corr }}(p)$ of ${ }^{4} \mathrm{He}$ is

$$
\begin{aligned}
& \eta^{\mathrm{corr}}(p)=\eta^{0}(p ; b)+\delta \eta\left(p ; b, c_{1}, y_{1}\right)+\delta \eta\left(p ; b,-c_{2}, y_{2}\right) \\
& -6 c_{1} c_{2} \frac{b^{3}}{\pi^{3 / 2}}\left[\frac{\mathrm{e}^{-p^{2} b^{2} \frac{1+2 y_{1}+2 y_{2}}{1+3 y_{1}+3 y_{2}+8 y_{1} y_{2}}}}{\left(1+3 y_{1}+3 y_{2}+8 y_{1} y_{2}\right)^{3 / 2}}-\frac{\mathrm{e}^{-p^{2} b^{2}}}{\left(1+2 y_{1}+2 y_{2}\right)^{3 / 2}}\right],
\end{aligned}
$$

where

$$
\delta \eta(p ; b, c, y) \equiv \frac{12 b^{3}}{\pi^{3 / 2}}\left[\frac{c^{2} \mathrm{e}^{-p^{2} b^{2} \frac{1}{1+2 y}}}{\left[(1+2 y)(1+4 y)^{3 / 2}\right.}-\frac{2 c \mathrm{e}^{-p^{2} b^{2} \frac{1+2 y}{1+3 y}}}{(1+3 y)^{3 / 2}}\right] .
$$

If we put $c_{2}=0$ in the above equations we end up with the corresponding expressions for the single gaussian $(1 \mathrm{G})$ correlation function, $f(r)=1-c \exp \left(-r^{2} / \beta^{2}\right)$ which has been used in evaluating several quantities of ${ }^{4} \mathrm{He}[26,27,28,29,31,32]$. We have introduced the $2 \mathrm{G}$ correlation function, that can overshoot unity at intermediate distances. This was found necessary in order to eliminate the negative values of $\eta_{2}\left(\vec{p}_{1}, \vec{p}_{2}\right)$ that result at some regions of intermediate values of momenta whenever a $1 \mathrm{G}$ correlation function is used.

The parameterization of the new, $2 \mathrm{G}$ correlation function by means of a rigorous procedure such as minimization and fitting to some set of experimental data (e.g. charge form factor data) lies beyond the scope of this work, therefore it was obtained somehow heuristically. First, it has been chosen to maintain the small- $r$ behaviour of the $1 \mathrm{G}$ correlation function of Ref. [31], by setting $c_{1}-c_{2}=0.8$ and $\beta_{1}=0.8 \mathrm{fm}$. Subsequently, the extra parameters have been determined requiring that the healing distance is equal to or somewhat lower than $1 \mathrm{fm}$ and the relative pair density distribution $\rho_{2}\left(r_{12}\right)$ is approximately zero at $r_{12}=0$. The value of the harmonic oscillator parameter $b$ was again chosen so as to reproduce the experimental charge root mean square radius of ${ }^{4} \mathrm{He}$. We ended up with $c_{2}=1.7$ (and therefore $c_{1}=0.9$ ), $\beta_{2}=1.1 \mathrm{fm}$ and $b=1.4 \mathrm{fm}$. In the left panel of Fig. 3 the resulting $2 \mathrm{G}$ correlation function, that overshoots unity, is plotted along with the $1 \mathrm{G}$ one of Ref. [31]. The corresponding values of a wound parameter $\kappa=\int\left(1-f\left(r_{12}\right)\right)^{2} \rho_{2}^{0}\left(r_{12}\right) \mathrm{d}^{3} r_{12}$ (where $\rho_{2}^{0}\left(r_{12}\right)$ is the relative pair density distribution function calculated in the harmonic oscillator model and normalized to unity) for the $2 \mathrm{G}$ and $1 \mathrm{G}$ correlations functions are equal to 0.008 and 0.018 respectively. In the center panel of the same figure the momentum distribution per particle $\eta(p) / A$ is plotted for both correlation functions, $2 \mathrm{G}$ and $1 \mathrm{G}$, along with the result of a variational calculation $[36,37]$. In the right panel of Fig. 3 the proton-proton relative pair density distribution (normalized to unity) $\rho_{p p}\left(r_{12}\right)$ is shown for both correlation functions $2 \mathrm{G}, 1 \mathrm{G}$. The bars cover the results of several variational calculations using a variety of two- and three-nucleon interaction models [38]. The values of $\eta(p)$ and $\rho_{p p}\left(r_{12}\right)$ calculated with the two-gaussian correlation function compare quite well with the ones of other methods.

Some numerical results for $\eta_{2}\left(\vec{p}_{1}, \vec{p}_{2}\right)$ are presented in Figs. 4 and 5 . In Fig. 4 , the $2 \mathrm{bMD}$ of ${ }^{4} \mathrm{He}$ is plotted in logarithmic scale, for $\vec{p}_{2}$ parallel to $\vec{p}_{1}, \vec{p}_{2}=\mathrm{p}_{2} \hat{p}_{1}$, as a function of $\mathrm{p}_{2}$, for $p_{1}=0,1,2,3 \mathrm{fm}^{-1}$. The correlated $2 \mathrm{bMD}$, as given by Eq. (25), is plotted with continuous lines and the harmonic-oscillator result, Eq. (23), with dashed lines. It seems that, within the present approximation, the correlated 2bMD shows deviations from 
the harmonic-oscillator picture mainly at high values of $p_{1}$ and/or $p_{2}$, developing highmomentum tails. In most cases, the deviations are larger when $\vec{p}_{1}, \vec{p}_{2}$ are antiparallel rather than parallel.

In Fig. 5 the quantity $\xi\left(\vec{p}_{1}, \vec{p}_{2}\right)$ for ${ }^{4} \mathrm{He}$ is plotted in logarithmic scale, as a function of $\cos \gamma$ for $p_{1}=p_{2}=2$ and $4 \mathrm{fm}^{-1}$. The continuous line includes SRC and the dashed line corresponds to the harmonic oscillator model and it equals 0.75 . Deviations from this value shows the effect of SRC. The correlated $\xi$ in the present approximation grows larger than unity at backward angles, and more so for large values of $p_{1}, p_{2}$, as indicated by the $p_{1}=p_{2}=4 \mathrm{fm}^{-1}$ graph. In this latter graph, deviations from 0.75 are large even at forward angles. We should note that for low values of $p_{1}$ and $p_{2}$ (not shown), the correlated $\xi$ takes values between about 0.7 and 1 .

We have also compared our results for the $2 \mathrm{bMD}$ with those obtained by using the single-gaussian correlation function of Ref. [31]. Although the overall behaviour of the $2 \mathrm{bMD}$ is not dramatically affected by the choice of $f(r)$, the two sets of results differ largely at certain kinematical regions, in particular at intermediate values of momenta where the single-gaussian correlation function yields negative results. (If the 2DM is approximated by only the first terms of the expression (22), i.e. $\rho_{2}^{\text {corr }}=f\left(r_{12}\right) f\left(r_{1^{\prime} 2^{\prime}}\right) \rho_{2}$, these regions of negative values show up as exact zeros at certain points [39].) As anticipated in Sec. 4.1, a similar, unphysical situation has been encountered in the study of other two-body quantities, when the LOA is used [27, 32]. It has been argued [32] that such regions indicate where the SRC start to show up dramatically, and are most sensitive to the approximation employed to account for the SRC. The results in these regions are also sensitive to the particular correlation function used. Let us note here that even in the case of the $2 \mathrm{G}$ correlation function a somewhat lower value of the oscillator parameter (eg. $b=1.35 \mathrm{fm}$ ) retrieves the negative values of the $2 \mathrm{bMD}$, indicating that the uncorrelated single-particle wavefunctions also play some role.

\section{Summary and Conclusions}

In summary, a compact analytical expression has been derived for the two-body momentum distribution, $\eta_{2}\left(\vec{p}_{1}, \vec{p}_{2}\right)$, of $Z=N$, $\ell$-closed nuclei within the context of the independentparticle shell model, using harmonic-oscillator wavefunctions. An application to the ${ }^{16} \mathrm{O}$ nucleus revealed interesting features arising from the Fermi statistics and the finite nuclear size. Next, the effect of short-range correlations was investigated by including Jastrowtype correlations in the calculation of $\eta_{2}\left(\vec{p}_{1}, \vec{p}_{2}\right)$ of the ${ }^{4} \mathrm{He}$ nucleus, using the low-order approximation of Ref. [27] and a two-gaussian correlation function. Significant deviations from the independent-particle picture were found for large values of $p_{1}$ and $p_{2}$ and for angles between the vectors $\vec{p}_{1}$ and $\vec{p}_{2}$ close to $\gamma=180^{\circ}$ and $0^{\circ}$. The quality of our approximation was also explored. It was found that, at certain regions of momenta, the results are sensitive to the correlation function used.

Using the same approximation, one could evaluate the 2bMD and investigate the effects of short-range correlations in other interesting nuclei, for example in ${ }^{16} \mathrm{O}$ and ${ }^{40} \mathrm{Ca}$. Furthermore, a general expression within the harmonic oscillator model applicable to every $\ell$-closed nucleus could be derived for the two-body momentum distribution $\eta_{2}\left(\vec{p}_{1}, \vec{p}_{2}\right)$, the two-body center-of-mass momentum distribution $\eta_{2}\left(\vec{P}_{\mathrm{CM}}\right)$ and the two-body relative momentum distribution $\eta_{2}\left(\vec{q}_{\text {rel }}\right)$, as well as for the two-hole spectral function $S\left(\vec{p}_{1}, \vec{p}_{2} ; E\right)$. The above calculations are relevant to the study of the two-nucleon knock-out reactions. 


\section{Acknowledgements}

Partial financial support from the University of Athens under Grant 70/4/3309 is acknowl-

edged. P.P. acknowledges a scholarship from the Greek State Scholarship Foundation (IKY).

\section{References}

[1] A.N. Antonov, P.E. Hodgson, and I.Zh.Petkov. Nucleon Correlations in Nuclei. Springer-Verlag, 1993; L.S. Cardman. Nucl. Phys. A654, (1999) 73c.

[2] L. Lapikas. Nucl. Phys. A553, (1993) 297c.

[3] V.R. Pandharipande, I. Sick, and P.K.A. de Witt Huberts. Rev. Mod. Phys. 69, (1997) 981.

[4] A.N. Antonov, P.E. Hodgson, and I.Zh. Petkov. Nucleon Momentum and Density Distributions in Nuclei. Claredon Press, Oxford, 1988.

[5] H. Müther and A. Polls. Prog. Part. Nucl. Phys. 45, (2000) 243.

[6] Proceedings of the Workshop on Two-Nucleon Emission Reactions, Elba International Physics Center, September 1989. Eds. O. Benhar and A. Fabrocini. ETS Editrice, Pisa, 1990.

[7] E. Jans. Proceedings of the Second Workshop on Electromagnetically Induced TwoNucleon Emission, Gent, May 17-20 1995, p.47.

[8] G. Rosner. Prog. Part. Nucl. Phys. 44, (2000) 99 and references therein.

[9] R. Starink et al. Phys. Lett. B474, (2000) 33.

[10] MAMI proposals, A1 collaboration, R. Neuhausen et al. A1/1-97, A1/4-98, A1/5-98.

[11] JLab proposals, E. Baghei et al. (spokespersons) PR-91-009, W. Bertozzi et al. (spokespersons) E-01-015.

[12] D.P. Watts et al. Phys. Rev. C62, (2000) 014616; C.J.Y.Powrie et al. Phys. Rev. C64, (2001) 034602.

[13] J. Aclander et al. Phys. Lett. B453, (1999) 211; A. Tang et al. nucl-ex/0206003, submitted to Phys. Rev. Lett.

[14] S. Boffi, C. Giusti, F.D. Pacati, and M. Radici. Electromagnetic Response of Atomic Nuclei. Claredon Press, Oxford, 1996.

[15] W.J.W. Geurts et al. Phys. Rev. C54, (1996) 1144.

[16] C. Giusti et al. Phys. Rev. C57, (1998) 1691.

[17] J. Ryckebusch et al. Nucl. Phys. A624, (1997) 581.

[18] M. Petraki, E. Mavrommatis, and J.W.Clark. Phys. Rev. C64, (2001) 024301.

[19] E. Mavrommatis, M. Petraki, and J.W. Clark. Phys. Rev. C51, (1995) 1849. 
[20] P. Papakonstantinou, E. Mavrommatis, and T.S. Kosmas. Nucl. Phys. A673, (2000) 171.

[21] O. Benhar and A. Fabrocini. Phys. Rev. C62, (2000) 034304.

[22] T.S. Kosmas and J.D. Vergados. Nucl. Phys. A510, (1990) 641.

[23] T.S. Kosmas and J.D. Vergados. Nucl. Phys. A536, (1992) 72.

[24] P. Papakonstantinou. Diploma thesis, University of Athens, 1995.

[25] M. Gaudin, J. Gillespie, and G. Ripka. Nucl. Phys. A176, (1971) 237.

[26] O. Bohigas and S. Stringari. Phys. Lett. 95B, (1980) 9.

[27] M. Dal Rì, S. Stringari, and O. Bohigas. Nucl. Phys. A376, (1982) 81.

[28] M.V.Stoitsov, A.N. Antonov, and S.S. Dimitrova. Phys. Rev. C48, (1993) 74.

[29] S.E. Massen and Ch.C.Moustakidis. Phys. Rev. C60, (1999) 024005.

[30] Ch.C. Moustakidis and S.E. Massen. Phys. Rev. C62, (2000) 034318.

[31] S.S. Dimitrova, D.N. Kadrev, A.N. Antonov, and M.V.Stoitsov. Eur.Phys.J. A7, (2000) 335 .

[32] G. Orlandini and L. Sarra. Proceedings of the Second Workshop on Electromagnetically Induced Two-Nucleon Emission, Gent, May 17-20 1995, p.1.

[33] Y. Haneishi and T. Fujita. Phys. Rev. C33, (1986) 260.

[34] S. Fantoni. Nucl. Phys. A363, (1981) 381.

[35] H. de Vries, C.W. de Jager, and C. de Vries. At. Dat. Nucl. Dat. Tables 36, (1987) 495.

[36] S.C. Pieper, R.B. Wiringa, and V.R. Pandharipande. Phys. Rev. C46, (1992) 1741.

[37] R.B. Wiringa. Phys. Rev. C43, (1991) 1585.

[38] J. Carlson et al. Phys. Rev. C38, (1988) 1879.

[39] P. Papakonstantinou, E. Mavrommatis, and T.S.Kosmas. Proceedings of the 11th Symposium of the Hellenic Nuclear Physics Society, Thessaloniki, Greece, 2000, to be published. 

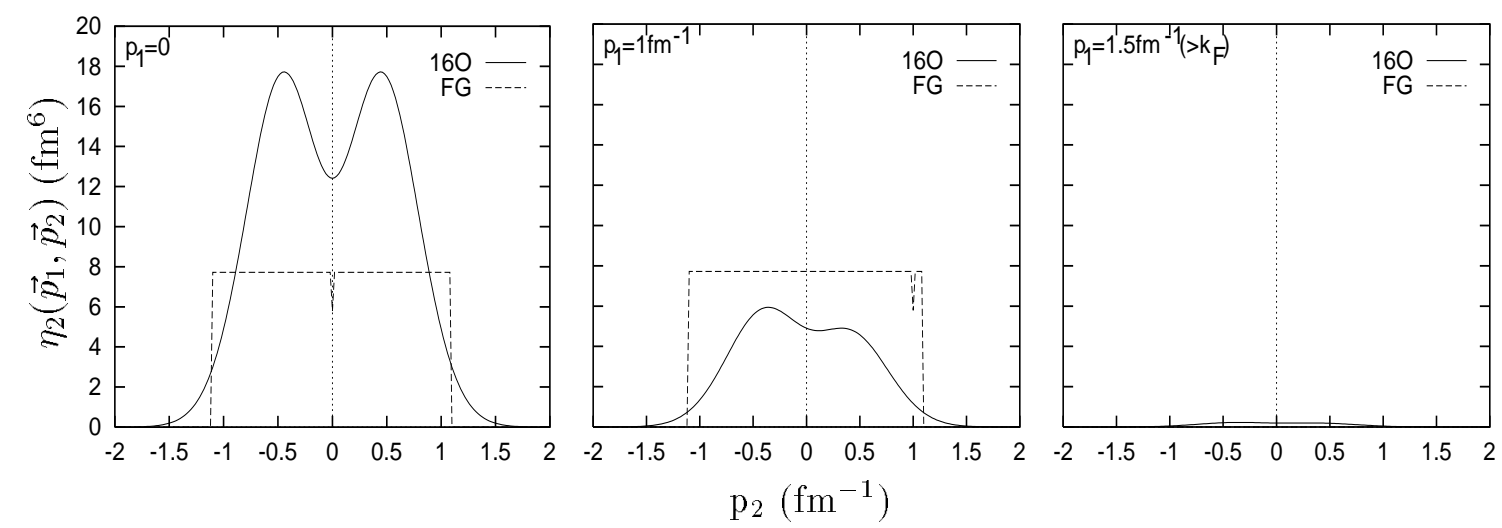

Figure 1: Two-body momentum distribution for $\vec{p}_{1}$ parallel to $\vec{p}_{2}$, as a function of $\mathrm{p}_{2}$ $\left(\vec{p}_{2}=\mathrm{p}_{2} \hat{p}_{1}\right)$ for $p_{1}=0,1,1.5 \mathrm{fm}^{-1}$, in the case of ${ }^{16} \mathrm{O}$ in the harmonic oscillator model (continuous line) and of an infinite ideal Fermi gas with $k_{\mathrm{F}}=1.1 \mathrm{fm}^{-1}$, normalized to the same number of particle pairs as in ${ }^{16} \mathrm{O}$ (dashed line).
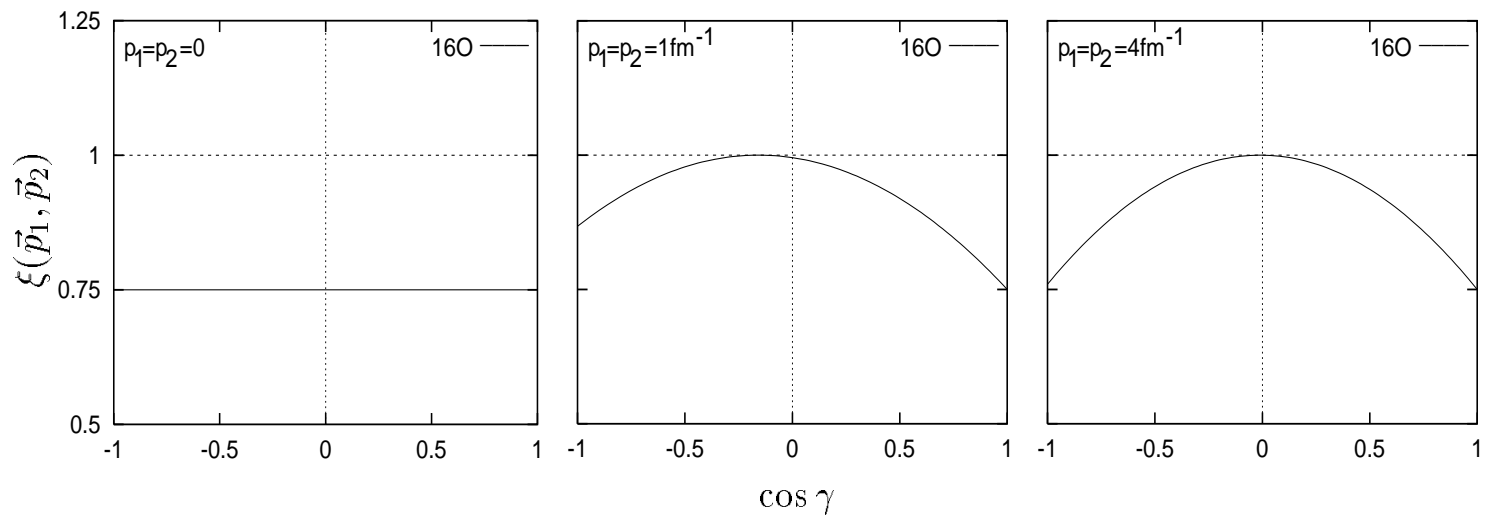

Figure 2: The quantity $\xi\left(\vec{p}_{1}, \vec{p}_{2}\right)$ (see Eq. (15)) as a function of $\cos \gamma(\gamma$ is the angle between the vectors $\vec{p}_{1}$ and $\left.\vec{p}_{2}\right)$ for $p_{1}=p_{2}=0,1,4 \mathrm{fm}^{-1}$, in the case of ${ }^{16} \mathrm{O}$ in the harmonic oscillator model (continuous line). 

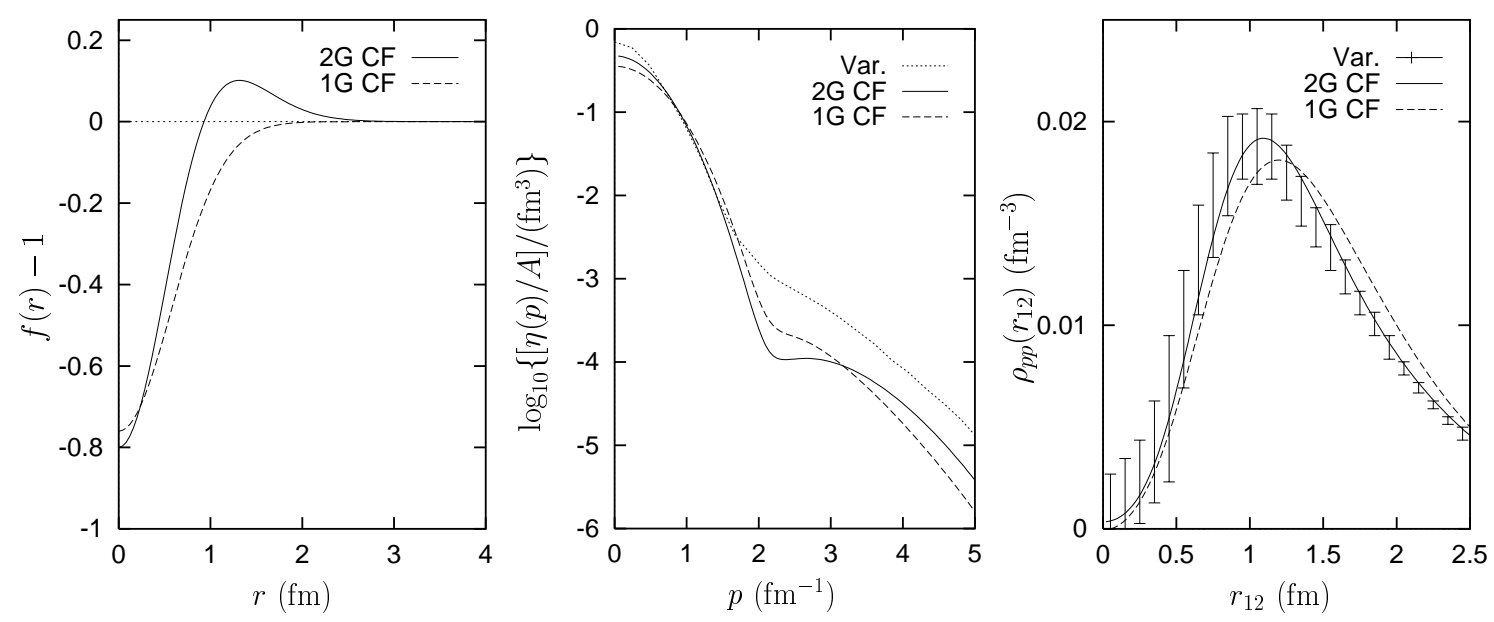

Figure 3: Left: The single-gaussian correlation function with $c=0.76, \beta=0.813 \mathrm{fm}$, from Ref. [31] (1G CF) and the two-gaussian correlation function employed in this work with the parameterization described in the text $(2 \mathrm{G} \mathrm{CF})$. Center: The momentum distribution per particle of ${ }^{4} \mathrm{He}$ calculated in LOA with the $1 \mathrm{G}$ and $2 \mathrm{G}$ correlation functions is compared with the variational calculation of Ref. [36] ("Var."). Right: The proton-proton relative pair density distribution (normalized to 1) of ${ }^{4} \mathrm{He}$ calculated in LOA with the $1 \mathrm{G}$ and $2 \mathrm{G}$ correlation functions is compared with the domain of results obtained via a set of variational calculations [38] ("Var."). 


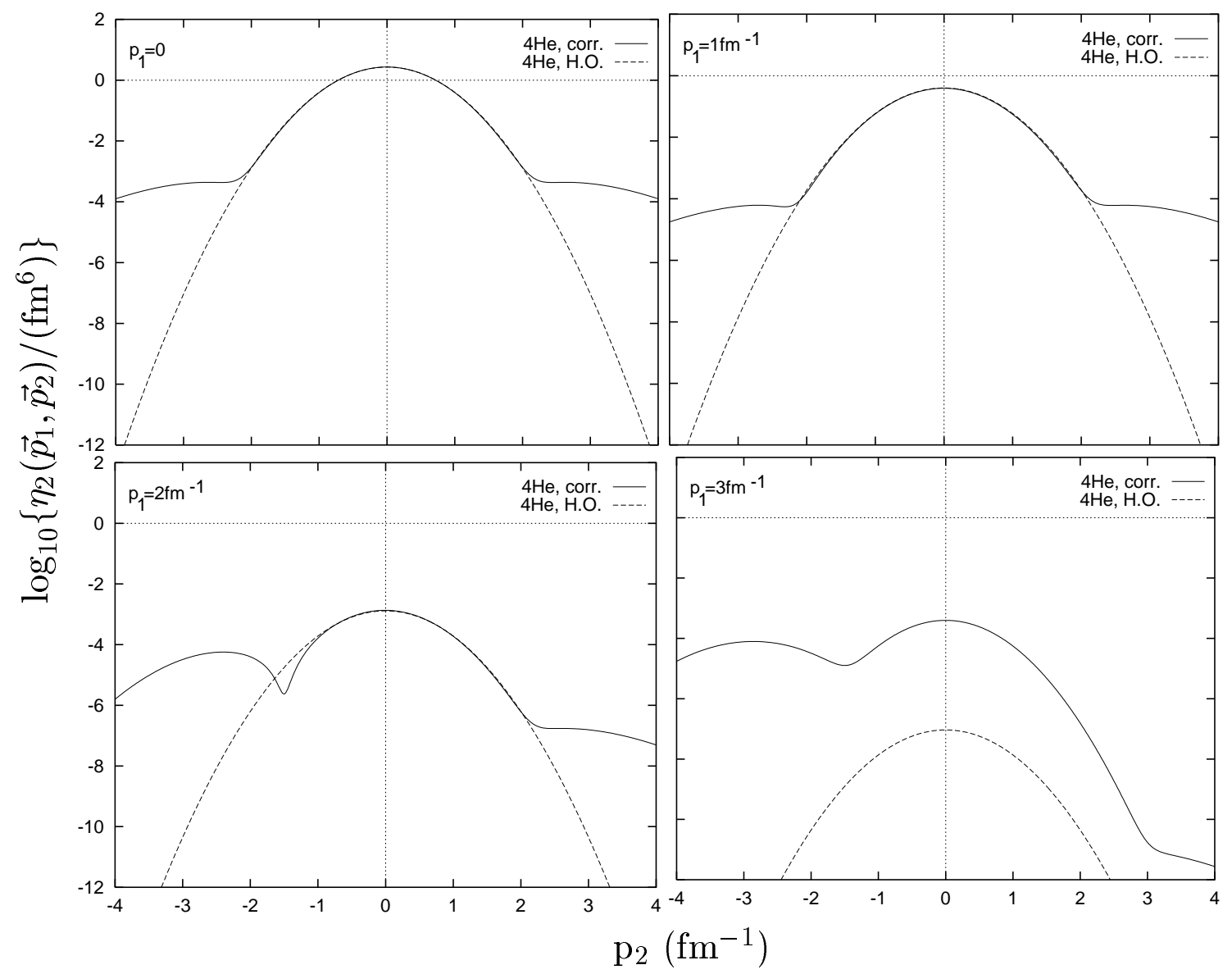

Figure 4: Two-body momentum distribution (in logarithmic scale) for $\vec{p}_{1}$ parallel to $\vec{p}_{2}$, as a function of $\mathrm{p}_{2}\left(\vec{p}_{2}=\mathrm{p}_{2} \hat{p}_{1}\right)$ for $p_{1}=0,1,2,3 \mathrm{fm}^{-1}$, in the case of ${ }^{4} \mathrm{He}$ including SRC, Eq. (25) (continuous line), and in the harmonic oscillator model, Eq. (23) (dashed line).

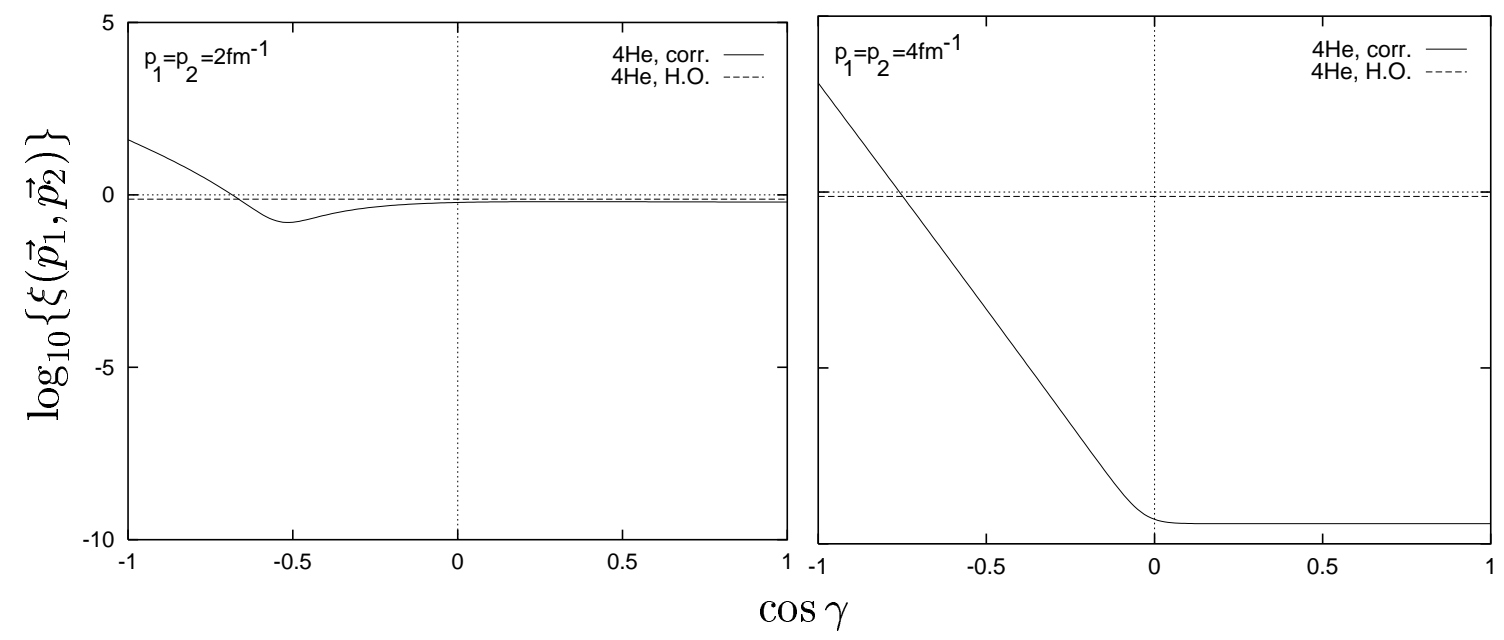

Figure 5: The quantity $\xi\left(\vec{p}_{1}, \vec{p}_{2}\right)$ (see Eq. (15)) in logarithmic scale, as a function of $\cos \gamma\left(\gamma\right.$ is the angle between the vectors $\vec{p}_{1}$ and $\left.\vec{p}_{2}\right)$ for $p_{1}=p_{2}=2,4 \mathrm{fm}^{-1}$, in the case of ${ }^{4} \mathrm{He}$ including SRC (continuous line), and in the harmonic oscillator model (dashed line). 